\title{
The relationship between network morphology and conductivity in nanotube films
}

\author{
Philip E. Lyons, ${ }^{1,2}$ Sukanta De, ${ }^{1,2}$ Fiona Blighe, ${ }^{1}$ Valeria Nicolosi, ${ }^{1}$ Luiz Felipe C. Pereira, ${ }^{1}$ \\ Mauro S. Ferreira, ${ }^{1}$ and Jonathan N. Coleman ${ }^{1,2, a)}$ \\ ${ }^{1}$ School of Physics, Trinity College Dublin, Dublin 2, Ireland \\ ${ }^{2}$ Centre for Research on Adaptive Nanostructures and Nanodevices (CRANN), Trinity College Dublin, \\ University of Dublin, Dublin 2, Ireland
}

(Received 10 March 2008; accepted 13 June 2008; published online 21 August 2008)

\begin{abstract}
We have characterized both the direct current conductivity and morphology of a wide range of films made from bundled nanotubes, produced by a selection of commercial suppliers. The conductivity increases with increasing nanotube graphitization but decreases with increasing film porosity $P$ and mean bundle diameter $\langle D\rangle$. Computational studies show that the network conductivity is expected to scale linearly with the number density of interbundle junctions. A simple expression is derived to relate the junction number density to the porosity and mean bundle diameter. Plotting the experimental network conductivities versus the junction number density calculated from porosity and bundle diameter shows an approximate linear relationship. Such a linear relationship implies that the conductivity scales quadratically with the nanotube volume fraction, reminiscent of percolation theory. More importantly it shows the conductivity to scale with $\langle D\rangle^{-3}$. Well-defined scaling with diameter and porosity allows the calculation of a specific conductivity expected for films with porosity of $50 \%$ and mean bundle diameter of $2 \mathrm{~nm}$. This predicted specific conductivity scales well with the level of nanotube graphitization, reaching values as high as $1.5 \times 10^{7} \mathrm{~S} / \mathrm{m}$ for well graphitized HiPCO single walled nanotubes. (C) 2008 American Institute of Physics.
\end{abstract}

[DOI: $10.1063 / 1.2968437]$

\section{INTRODUCTION}

Much work is underway at present to develop thin, flexible films of carbon nanotubes (NTs) for applications such as transparent electrodes. ${ }^{1-7}$ Such films should display high transparency coupled with low sheet resistance. The relationship between transparency and sheet resistance for thin conducting films is controlled by the ratio of direct current conductivity $\left(\sigma_{\mathrm{dc}}\right)$ to optical conductivity $\left(\sigma_{\mathrm{op}}\right),{ }^{8}$ such that high values of $\sigma_{\mathrm{dc}} / \sigma_{\mathrm{op}}$ lead to the required properties. The value of $\sigma_{\mathrm{op}}$ for typical NT thin films depends on the film density and tends to be close to $1.7 \times 10^{4} \mathrm{~S} / \mathrm{m} @ 550 \mathrm{~nm} .{ }^{9}$ Thus, in order to optimize the properties of transparent electrodes, the dc conductivity of NT films must be maximized. Currently, the best conductivities reported have been $\sim(5-6)$ $\times 10^{5} \mathrm{~S} / \mathrm{m}^{7,10}$ However, industry specifications for transparent electrodes require transparencies and sheet resistances better than $90 \%$ and $90 \Omega / \square$, respectively. To achieve this, we can calculate that the film conductivity must exceed 7 $\times 10^{5} \mathrm{~S} / \mathrm{m}^{11}$ Thus, procedures must be developed to improve the conductivity of even the best NT films. A number of chemical routes have been demonstrated to achieve this, namely, via chemical doping ${ }^{12}$ and functionalization. ${ }^{13}$ However, NT films with conductivity exceeding $7 \times 10^{5} \mathrm{~S} / \mathrm{m}$ have not been reported as yet. In order to produce such conductive films, it will be necessary to fully understand the factors controlling the conductivity of NT films.

Unlike the optical conductivity, the dc conductivity is limited by the tunneling of electrons from tube to tube (or

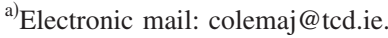

more realistically from bundle to bundle). Thus the overall dc conductivity will depend critically on the number of conductive paths through the film and the typical number of interbundle junctions encountered on a given path. Thus, these parameters, and so the dc conductivity, are intimately linked to the network morphology. Very little work has been done on the effect of the morphological properties of the NT films on the conductivity. NT films are always porous and always consist of networks of bundles, not individual NTs. The porosity tends to vary from film to film as does the bundle size. Thus, any attempt to maximize the dc conductivity must be preceded by optimization of the morphological properties of the film.

In this paper we explore whether the conductivity is solely limited by the inter-NT junction resistance or if it is affected by the properties of the NTs themselves. In addition, we attempt to determine how the network properties affect the ultimate film conductivity. By measuring bulk dc conductivity of NT films made from a range of different tube types, we demonstrate that the film conductivity increases with increasing NT graphitization. In addition, the conductivity decreases both with increasing film porosity and with increasing bundle size. Using experiment and computer modeling, we show that the conductivity scales approximately with the inter-NT junction density that we derive in terms of porosity and bundle diameter. Finally, we introduce the "specific conductivity," which we show scales with the Raman $G / D$ ratio, emphasizing that the properties of the NTs themselves and not just the network properties are important for high conductivity films. 
TABLE I. Commercial NT suppliers and the NT types used in this work. Also shown are the NT purity as given by the suppliers' website and the solvents used. NB the purity quoted is the fraction of the carbon in the material in the form of NTs.

\begin{tabular}{|c|c|c|c|}
\hline Supplier & NT type (functional groups in brackets) & Carbon purity $(\%)$ & Solvent \\
\hline Carbon NanoTechnologies Inc. & HiPCO SWNTs & $>90$ & NMP,DMF \\
\hline \multirow[t]{2}{*}{ Nanocyl } & CCVD SWNTs & $>70$ & NMP \\
\hline & CCVD DWNTs & $>90$ & NMP \\
\hline Elicarb, Thomas Swan and & As prepared CVD SWNTs & $>70$ & NMP \\
\hline Co. Ltd & Annealed CVD SWNTs & $>90$ & NMP \\
\hline SouthWest & CoMoCAT® SWNTs & $>90$ & NMP \\
\hline \multicolumn{4}{|l|}{ NanoTechnologies Inc. } \\
\hline Nanolab & CVD DWNTs & $\cdots$ & NMP \\
\hline \multirow[t]{6}{*}{ Carbon Solutions Inc. } & As prepared arc discharge SWNTs & $60-85$ & NMP \\
\hline & P2-SWNT (low level-COOH) & $80-100$ & NMP,DMF \\
\hline & P3-SWNT (high level-COOH) & $80-100$ & NMP,DMF \\
\hline & P5-SWNT (octadecylamine) & $85-95$ & Chloroform \\
\hline & P7-SWNT (polyethyleneglycol) & $85-95$ & $\mathrm{H}_{2} \mathrm{O}$ \\
\hline & P8-SWNT (m-polyaminobenzene sulfonic acid) & $85-95$ & $\mathrm{H}_{2} \mathrm{O}$ \\
\hline
\end{tabular}

\section{EXPERIMENTAL PROCEDURE}

Different types of single wall NTs (SWNTs), double wall NTs (DWNTs), and functionalized SWNTs were purchased from six commercial producers, as shown in Table I, and used as supplied. Dispersions of NTs were prepared in different solvents ${ }^{14,15}$ (Table I) by stepwise sonication using a high power ultrasonic tip processor model GEX600 (120 W and $60 \mathrm{kHz}$ ) and a low-power ultrasonic bath (model Ney Ultrasonic). These dispersions were produced at a starting concentration of $0.444 \mathrm{mg} / \mathrm{ml}$ in the appropriate solvent by sonicating for 3 min using the ultrasonic tip processor. The initial dispersions were then diluted to a concentration of $0.08 \mathrm{mg} / \mathrm{ml}$ and subjected to another minute of high power tip sonication, followed by $4 \mathrm{~h}$ in a low-power ultrasonic bath. Finally the dispersions were sonicated by ultrasonic tip processor for 0-15 min, depending on the tube type. In contrast to the other NT types, films from HiPCO SWNT in $\mathrm{N}$-methyl-pyrrolidone (NMP) were prepared using dispersions sonicated at a range of sonication times: $0,2,4$, and 6 min. NT films were prepared by filtering $150 \mathrm{ml}$ of each dispersion through a porous polyvinylidene fluoride (PVDF) filtration membrane (Millipore, Durapore membrane filters, $0.45 \mu \mathrm{m}$ pore size). The deposited CNT films were washed with $250 \mathrm{ml}$ of de-ionized water followed by $50 \mathrm{ml}$ of acetone and dried under vacuum at room temperature for $12 \mathrm{~h}$. The dried films, with an average thickness of $20 \mu \mathrm{m}$, were peeled off the filter membrane and cut into strips with an average width of $2.25 \mathrm{~mm}$ for detailed characterization.

The resistance was measured as a function of strip length by a two probe method. ${ }^{16}$ This is done by applying painted silver electrodes to the ends of a strip, measuring the resistance, cutting the end off, reapplying the electrode, and remeasuring the resistance. This was repeated a number of times to give a set of eight to ten resistance/length values. The graphitization of the CNTs after processing into films was investigated using Raman spectroscopy with excitation wavelength of $633 \mathrm{~nm}$ (Horiba Jobin Yvon, LabRam HR Series). For each film, Raman measurements were made on five different regions of the films. In all cases these spectra were similar for a given film. The five spectra were then averaged to give a final representative Raman spectrum. The film density was measured by carefully weighing the films using a microbalance and measuring the dimensions using a digital micrometer. The porosity of the films was calculated using the measured density of the films and the accepted density of SWNTs $\left(1500 \mathrm{~kg} / \mathrm{m}^{3}\right)$. The average bundle diameters of the deposited films were measured using field emission scanning electron microscopy (SEM) (Hitachi S-4300) operated with an acceleration of $5 \mathrm{kV}$ (resolution $\sim 4 \mathrm{~nm}$ ). In all cases the mean diameters were significantly larger than the instrumental resolution.

\section{RESULTS}

\section{A. Film characterization}

dc conductivity measurements were made for all NT films. The bulk conductivity was calculated by measuring the film resistance as a function of film length. ${ }^{16}$ The advantage of this method is that it results in a well defined potential in these (relatively thick and in some cases relatively resistive) films, guaranteeing current flow throughout the bulk of the film. In all cases the resistance scaled linearly with film length as predicted by Ohm's law. The conductivity is then obtained from the slope of the resistance/length curve while the contact resistance is obtained from the intercept. In all cases the contact resistance was close to zero. The conductivity of the films ranged from $2.08 \times 10^{2} \mathrm{~S} \mathrm{~m}^{-1}$ for the film of Nanocyl SWNT dispersed in NMP up to 1.67 $\times 10^{4} \mathrm{~S} \mathrm{~m}^{-1}$ for the film of HiPCO tubes dispersed in dimethyl-formamide (DMF). Notably, the carboxylic acid functionalized P3 SWNTs (Carbon Solutions Inc.) dispersed in DMF displayed a reasonably high conductivity (1.3 $\times 10^{4} \mathrm{~S} \mathrm{~m}^{-1}$ ) despite their high levels of functionalization. This is probably because their $-\mathrm{COOH}$ functionalities are thought to be predominately at the tube ends ${ }^{17}$ and so have limited effect on charge transport through the tube bodies.

Skakalova et al. ${ }^{18}$ reported the conductivity for Buckypaper prepared from HiPCO SWNT dispersed in sodium dodecyl sulfate solution to be $5.5 \times 10^{4} \mathrm{~S} \mathrm{~m}^{-1}$, quite similar to our result for the film of HiPCO dispersed in DMF. How- 


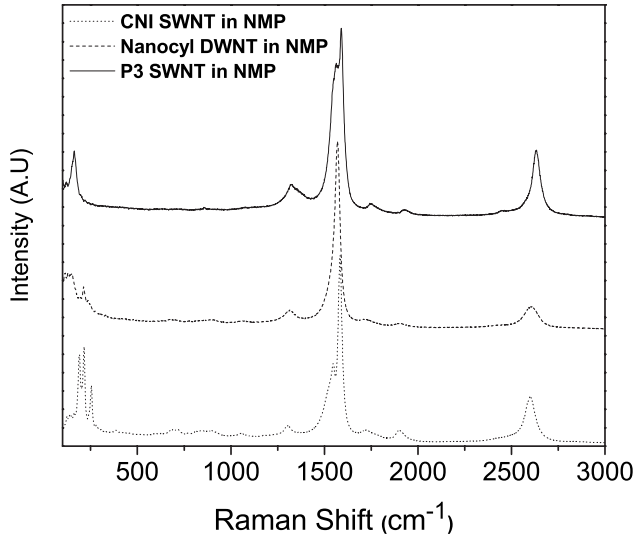

FIG. 1. Typical Raman spectra for three of the film types used in this study.

ever, much higher values for conductivity of thin NT films, of up to $6 \times 10^{5} \mathrm{~S} \mathrm{~m}^{-1}$, have been reported. ${ }^{7}$ The discrepancy between this value and the values presented here is most likely due to differences in film morphology as will be discussed below.

Two main factors control charge transport through NT films, the conductivity of the tubes themselves and the ability of carriers to tunnel from tube to tube. To simplify matters, we neglect variations in metal/semiconductor populations and ignore the effects of slightly different diameter distributions for different tube types. Within these approximations, the conductivity of the NTs themselves is controlled by the defect population associated with a given tube type. ${ }^{19} \mathrm{We}$ justify this assumption by noting that in all cases the networks are made up of bundles not individual NTs. Current flow in bundles is thought to be predominately through the metallic NTs. We expect that the effective conductivity of these metallic tubes is strongly effected by their defect content. ${ }^{19}$ The rate of tunneling across a given bundle-bundle junction is of course controlled by the junction width and the electronic properties of the constituent tubes and so the mean tunneling rate is controlled by the statistics of the distribution of junctions. This is not something that is easy to control for a given sample of NTs. However, the number of junctions that a carrier must traverse, on average, when traveling through a NT film depends on how the NTs/bundles are arranged within the film, i.e., the network morphology. For our purposes, we can describe the film morphology using two parameters, the porosity and the mean bundle diameter. These parameters then determine the junction population in these networks. Thus, in order to investigate the factors controlling the conductivity of NT films we quantify the porosity and the mean bundle diameter by density measurements and SEM, respectively. In addition, we probe the level of defects in each tube type by Raman spectroscopy.

Raman spectroscopy is one of the most widely used techniques to characterize SWNTs. Shown in Fig. 1 are the Raman spectra of three selected films, each displaying a number of key features, including the breathing modes of the NTs $\left(\sim 200 \mathrm{~cm}^{-1}\right)$, the $D$ band $\left(\sim 1300 \mathrm{~cm}^{-1}\right)$, the $G$ band $\left(\sim 1570 \mathrm{~cm}^{-1}\right)$, and the $D^{*}$ band $\left(\sim 2600 \mathrm{~cm}^{-1}\right)$. For our purposes we are interested in the $G$ and $D$ bands. The relative intensity of the former is generally associated with the

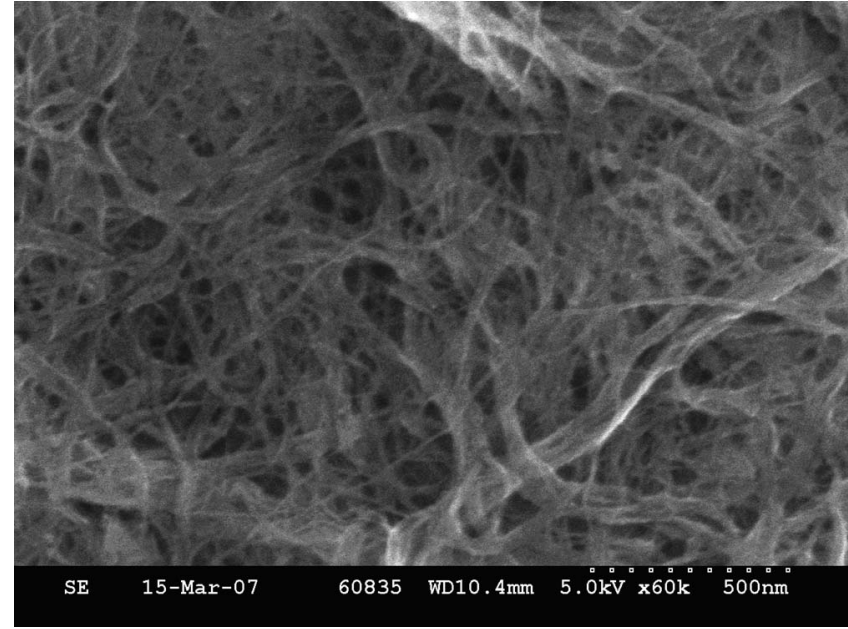

FIG. 2. SEM image of the surface of a film prepared from HiPCO NTs in NMP. This image is representative of all the NT films made in this study.

level of perfection of the hexagonal graphitic lattice. In contrast, for samples containing only NTs, the relative intensity of the $D$ band is related to the presence of disorder in the tube. However, unpurified NT samples also have a contribution to the $D$ band from non-NT carbonaceous impurities. In all cases, the tubes used in this work have been purified by the manufacturer. The quoted purity values are given in Table I, where available, and in the vast majority of cases exceed $80 \%$. While it is prudent to treat manufacturers purity figures with caution, examination of SEM images have not shown significant quantities of impurities in any case. Thus we associate the $D$ band of our Raman spectra with imperfections to the hexagonal lattice of the NTs. Then, by measuring the ratio between the $G$-band and the $D$-band intensities $I_{G} / I_{D}$ we have an approximate metric for the perfection of the hexagonal lattice. These ratios varied from 19.5 for well graphitized tubes (South Western NanoTechnologies Inc. SWNTs in NMP) down to 3.2 for very defective tubes (P7 SWNTs in $\mathrm{H}_{2} \mathrm{O}$ ).

The average bundle diameters in the films were measured using SEM. A scanning electron micrograph for the film of HiPCO SWNT prepared using NMP based dispersions is shown in Fig. 2. This image is typical of NT films in general. The mean bundle diameter for this sample was $\sim 18 \mathrm{~nm}$. The average bundle diameter varied between 10 $\mathrm{nm}$ (P3 SWNTs in DMF) and $21 \mathrm{~nm}$ (P2 SWNTs in NMP). As can be clearly seen from Fig. 2, NT based films tend to be quite porous. ${ }^{20}$ We have calculated the porosity of the films $P$ from the density of films $\rho_{\text {film }}$ and the density of CNT $\rho_{\mathrm{NT}}$ using $^{20} P=1-\rho_{\text {film }} / \rho_{\mathrm{NT}}$. The porosity of the SWNT films varied greatly from $42 \%$ (South Western Nanotechnologies Inc. SWNT in NMP) to $76 \%$ (Nanocyl SWNT in NMP). The porosity of the functionalized SWNTs exhibited a smaller variation, ranging between 48\% (P3 SWNTs in NMP) and $66 \%$ (P7 SWNTs in water). It should be noted that the porosity is of course related to the NT volume fraction by $V_{f}$ $=(1-P)$. We shall use both parameters in this paper.

As discussed above, the conductivity of CNT films should be dependent on two main physical aspects, the NT structure (graphitization) and the film morphology. Using the 

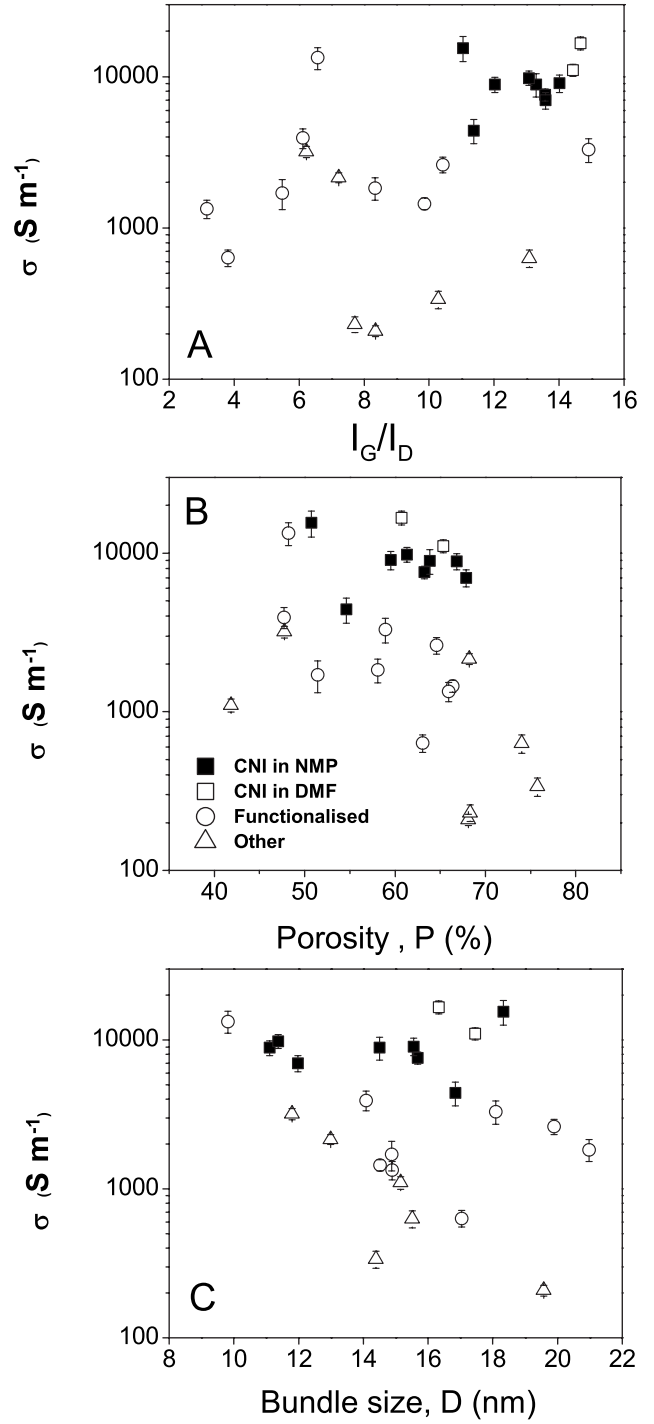

FIG. 3. Dependence of film conductivity on both NT properties and network properties. (a) Film conductivity as a function of Raman ratio showing scaling of conductivity with NT graphitization. (b) Film conductivity as a function of film porosity showing a decrease in conductivity for more porous films. (c) Film conductivity as a function of bundle diameter showing higher conductivity for more exfoliated films.

ratio of Raman $G$-band to $D$-band intensities as a proxy for structural perfection, we plot the film conductivity as a function of $I_{G} / I_{D}$ in Fig. 3(a). While the data are quite scattered, it is clear that the conductivity tends to be larger for films made from tubes with higher $I_{G} / I_{D}$, ie, higher structural perfection. This is unsurprising as the conductivity of individual CNTs has been shown ${ }^{19}$ to be heavily dependent on the presence of defects in the graphitic structure. These defects can include pentagons, heptagons, vacancies, dopant, or functional groups that all act to alter the electronic structure of the perfect tubes.

The film morphology can be characterized by the porosity and the average bundle diameter. We plot the film conductivity as a function of porosity in Fig. 3(b). While the data display some scatter, the trend of decreasing conductivity with increasing porosity is clear. Qualitatively, this trend makes sense as a sparser network (more porous) would certainly be expected to be less conductive. Similarly we plot conductivity versus mean bundle diameter in Fig. 3(c). Again, while some scatter is observed, the trend is clear with the conductivity decreasing steadily as the bundle diameter increases. This latter phenomenon is not unexpected. Hecht et $a l^{21}$ recently predicted that the conductivity of thin NT film should scale approximately as $1 / D^{2}$, a result based on an analysis of the dependence of the number of junctions in a system on the level of bundling.

\section{DISCUSSION}

\section{A. Interbundle junction density and bundle connectivity}

However, any more detailed analysis of the conduction process would have to consider the number of conducting pathways through the film. This is less than straightforward and would require detailed knowledge of the arrangement of bundles within the film. However we can postulate that the number of conductive paths is related to the number density of inter-NT junctions. This is a quantity that is much easier to calculate. We can easily estimate the number of junctions per unit volume of film $N_{J}$ by calculating the number of bundles per unit volume and multiplying by half the mean number of junctions per bundle $\langle\alpha\rangle / 2$. The number of bundles per unit volume is related to the film density and the mean bundle diameter $\langle D\rangle$ giving

$$
N_{J}=\frac{\langle\alpha\rangle}{2} \frac{\rho_{\mathrm{film}} / \rho_{\mathrm{NT}}}{\pi\langle D\rangle^{2}\langle L\rangle / 4}=\langle\alpha\rangle \frac{2(1-P)}{\pi\langle D\rangle^{2}\langle L\rangle},
$$

where $\langle L\rangle$ is the mean bundle length and $\langle\alpha\rangle$ is the mean number of junctions per bundle. Using $\langle\alpha\rangle / 2$ accounts for double counting of junctions.

Estimating $\langle\alpha\rangle$ is slightly less straightforward. We proceed by considering a cylindrical shell of thickness $\langle D\rangle$ surrounding a test bundle (diameter $\langle D\rangle$ ). We then estimate how many bundles (diameter $\langle D\rangle$ ) intersect this shell under circumstances similar to those within a NT network. We begin by estimating the volume of intersection of an arbitrary bundle with the shell. While the volume of intersection for two cylinders (diameters $\langle D\rangle$ and $3\langle D\rangle$ ) under these circumstances $(\langle D\rangle<$ cylinder axis separation $\langle 2\langle D\rangle)$ can be calculated, the result is not a simple analytic function. ${ }^{22}$ However it can be shown numerically that the volume of intersection scales with $\langle D\rangle^{3}$. We assume that the mean volume of intersection is just $V_{\text {int }}=\kappa\langle D\rangle^{3}$, where $\kappa$ is a constant that we assume to be invariant with bundle diameter (and film porosity). We can use the measured density of the film to estimate the total mass (associated with intersecting cylinders) contained within the shell described above to be $\approx \rho_{\text {film }} 8\langle D\rangle^{2}\langle L\rangle \pi / 4$. This mass is localized in the volumes of intersection described above. This allows us to estimate the number of bundles intersecting a given shell to be equal to the total mass within the shell divided by the mass per volume of intersection $\left(M_{\mathrm{int}}=\rho_{\mathrm{NT}} \kappa\langle D\rangle^{3}\right)$. In a previous paper ${ }^{23}$ we made the approximation that every bundle that intersects the shell is in contact with our test bundle. This means that the number of junctions per bundle is just the number of bundles within the shell and so 


$$
\langle\alpha\rangle \approx \frac{\rho_{\mathrm{film}}}{\rho_{\mathrm{NT}}} \frac{8 \pi\langle L\rangle}{4 \kappa\langle D\rangle}=(1-P) \frac{2 \pi\langle L\rangle}{\kappa\langle D\rangle} .
$$

However not all bundles intersecting the shell are actually in contact with the test bundle. To account for this, we assume (see below) that Eq. (2) is correct within a constant factor. This allows us to rewrite Eq. (2) as

$$
\langle\alpha\rangle \approx \beta(1-P) \frac{\langle L\rangle}{\langle D\rangle},
$$

where we consolidate all constants in a single constant, $\beta$.

To test the veracity of this approach we carried out numerical simulations to model the connectivity of a conducting network formed by a large number of rods. Because the network is inherently disordered, we must account for fluctuations in the network connectivity by considering a truly disordered network made of randomly distributed finite-sized one-dimensional rods contained within a box of side $2 L$ $\times 2 L \times 4 L$, where $L$ is the rod length. The rods are assumed to be identical with diameter $D$, length $L$ and aspect ratio (AR) defined as $\mathrm{AR}=L / D$. The volume fraction of rods $V_{f}$ is related to the porosity $P$ by $V_{f}=1-P$ and is easily obtained by calculating the volume occupied by the rods within a sampling cubic box of arbitrary size. The connectivity of the network can be calculated by looking at the shortest distance between rods within the sampling box. If the shortest distance between two rods is less than the rod diameter, we assume them to be connected. By counting over all possible pairs within the box we can map the connectivity of the network. This approach allows us to calculate (among other things) the number of junctions per rod $\langle\alpha\rangle$. It should be noted that statistical significance is only achieved after averaging over several configurations of disorder which allows calculation of the dispersions in the volume fraction and in the mean number of connections per rod, respectively.

As predicted by Eqs. (2) and (3), the calculated value of $\langle\alpha\rangle$ increases linearly with the volume fraction $V_{f}$, with a rate of increase which depends on the AR of the rods [see Fig. 4(a))]. When plotted as a function of the product $V_{f} \times L / D$ [Fig. 4(a) inset], $\langle\alpha\rangle$ grows linearly along the same straight line for all aspect ratios, confirming the veracity of Eq. (3). By comparison of the measured slope of Fig. 4(a) (inset) with Eq. (3), we can estimate $\beta \approx 1.93$. We can use the calculated value of $\beta$ to show that $\langle\alpha\rangle$ ranges from 54 to 124 junctions/um of bundle length for the P2-NMP and CNINMP samples, respectively. It should also be pointed out that $\beta$ (and hence $\kappa$ ) is invariant with bundle diameter and volume fraction.

Combining Eqs. (1) and (3) we get an expression for the number of junctions per unit volume

$$
N_{J} \approx \frac{2 \beta}{\pi} \frac{(1-P)^{2}}{\left\langle D^{3}\right\rangle}=\frac{2 \beta}{\pi} \frac{V_{f}^{2}}{\left\langle D^{3}\right\rangle},
$$

where we remember that $(1-P)=V_{f}$, the NT volume fraction. We can calculate the junction number density to vary from $1.8 \times 10^{22} \mathrm{~m}^{-3}$ for the Nanocyl SWNT in NMP to $3.7 \times 10^{23} \mathrm{~m}^{-3}$ for the P3 SWNT in DMF.
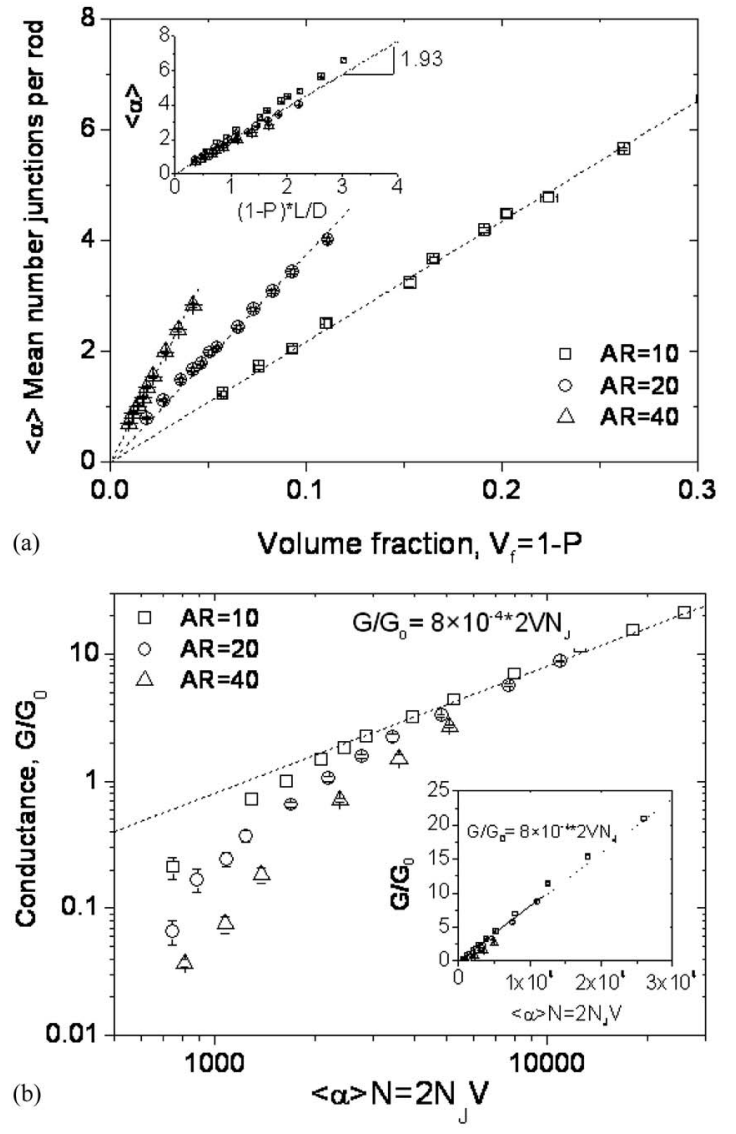

FIG. 4. Computer simulations of properties of random networks of rods. (a) Calculations of mean number of junctions per rod $\langle\alpha\rangle$ as a function of volume fraction for three different ARs. Inset: mean number of junctions per rod as a function of volume fraction times AR showing universal scaling as predicted by Eq. (3). (b) Calculations of the conductance of a network of $N$ rods as a function of $\langle\alpha\rangle N$, for three different ARs. $G$ is expressed in units of $\left(G_{0}\right)$ where $G_{0}$ is the junction conductance. The fact that all curves asymptotically approach the dashed line (linear scaling) shows that the conductance scales with the number of junctions per unit volume $N_{J}$ where $N_{J}$ $=\langle\alpha\rangle N / 2 V$, with $V$ as the volume of the box containing the rods. Inset: The same data shown on a linear-linear plot. The dotted line represents $G / G_{0}$ $=8 \times 10^{-4} 2 N_{J} \mathrm{~V}$

\section{B. Relationship between network conductivity and $\boldsymbol{N}_{J}$}

As discussed above, we expect the network conductivity to depend on both the NT conductivity and the network morphology. However, if the NTs themselves are much less resistive than the junctions we might expect that the conductivity to be dominated by the network properties. In this scenario, the conductivity would be expected to scale approximately with $N_{J}$.

Again we can use numerical simulation to investigate the relationship between the network conductivity and the number of junctions per unit volume $N_{J}$. Having established how the average connectivity scales with the volume fraction, we proceed to investigate how the conductance, G, of our disordered network scales with the mean number of connections per rod $\langle\alpha\rangle$. When calculating $\langle\alpha\rangle$ [Fig. 4(a)], we are able to map the connectivity of the network and identify not only which tubes are connected to which but also how they are distributed across the film. By enclosing this network in a sampling box whose sizes exceed the NT length, we are also able to estimate the number of tubes that intersect two oppo- 
site surfaces of the box (these surfaces play the role of electrodes). Again, the box size used was $2 L \times 2 L \times 4 L$ with the electrodes on opposing $2 L \times 2 L$ faces, where $L$ is the rod length. With this connectivity map, we consider a large number of conducting NTs and model them as nodes joined by resistors of resistance $R_{0}$ (conductance $G_{0}=1 / R_{0}$ ), which physically represent the barrier experienced by the charge carriers to hop between neighboring tubes. Once the network is defined we can calculate the equivalent resistance between any two arbitrary nodes as well as the corresponding conductance. These calculations were carried out for boxes with different numbers of nodes and hence different numbers of NTs, $N$. Due to constraints in computational power, the largest calculation carried out was for a number of rods $N$ $=1860$ such that $\langle\alpha\rangle N$ was 26000 . Furthermore, in order to achieve statistical significance, all results were obtained after averaging over several configurations of disorder.

The calculations above show that, for each value of rod $\mathrm{AR}$, the conductance increases monotonically with $\langle\alpha\rangle$ (not shown). To understand the relationship between the conductivity, as represented here by the network conductance and the number of junctions per volume $N_{J}$, we can replot the conductance as a function of $N_{J}$ by noting the relationship

$$
N_{J}=\frac{\langle\alpha\rangle}{2} \frac{N}{V},
$$

where $N / V$ is the total number of rods per unit volume. The conductance is plotted in Fig. 4(b) as a function of $\langle\alpha\rangle N$ $=2 N_{J} V$ for three different rod ARs. For each AR, the conductance increases with increasing $\langle\alpha\rangle N$. While each curve is independent at low values of $\langle\alpha\rangle N$, they come together for values of $\langle\alpha\rangle N \approx 5000$ and subsequently appear to scale with the same linear trend. As the box volume $V$ was constant in all cases, this strongly suggests that, for values of $\langle\alpha\rangle N$ $>5000$, the network conductance scales linearly with $N_{J}$. Due to restrictions of computation time, we were unable to compute data points with large enough $\langle\alpha\rangle N$ to show unambiguously, that the data for each AR converge on the same straight line (calculations with $\mathrm{AR}=40$ are more time consuming). However by plotting the high AR data on a linearlinear curve [Fig. 4(b), inset], we see that the curves for each AR increase linearly with similar slopes, $G / G_{0}=8 \times 10^{-4}$ $\times 2 V \mathrm{~N}_{J}$. For the experimental NT films, we measured the lowest value of $N_{J}$ to be $\sim 1.8 \times 10^{22} \mathrm{~m}^{-3}$. Coupled with the size of the box used in the computation (taking $L>1 \mu \mathrm{m}$ ) we can estimate a realistic lower value for a network of $\langle\alpha\rangle N=2 N_{J} V$ to be $>3 \times 10^{5}$, well into the region where we expect the data to have coalesced on the same straight line. Thus, in any real network, we can be confident that $G / G_{0}$ $\propto N_{J}$ for all ARs.

This linear relationship between the network conductance $G$ and $N_{J}$ allows us to write (with reference to Eq. (4))

$$
\sigma \approx k \frac{(1-P)^{2}}{\left\langle D^{3}\right\rangle}=k \frac{V_{f}^{2}}{\left\langle D^{3}\right\rangle},
$$

where $k$ is a constant for a given tube type. Note that this expression scales as $1 /\left\langle D^{3}\right\rangle$ not $1 /\left\langle D^{2}\right\rangle$ as suggested previously. $^{21}$
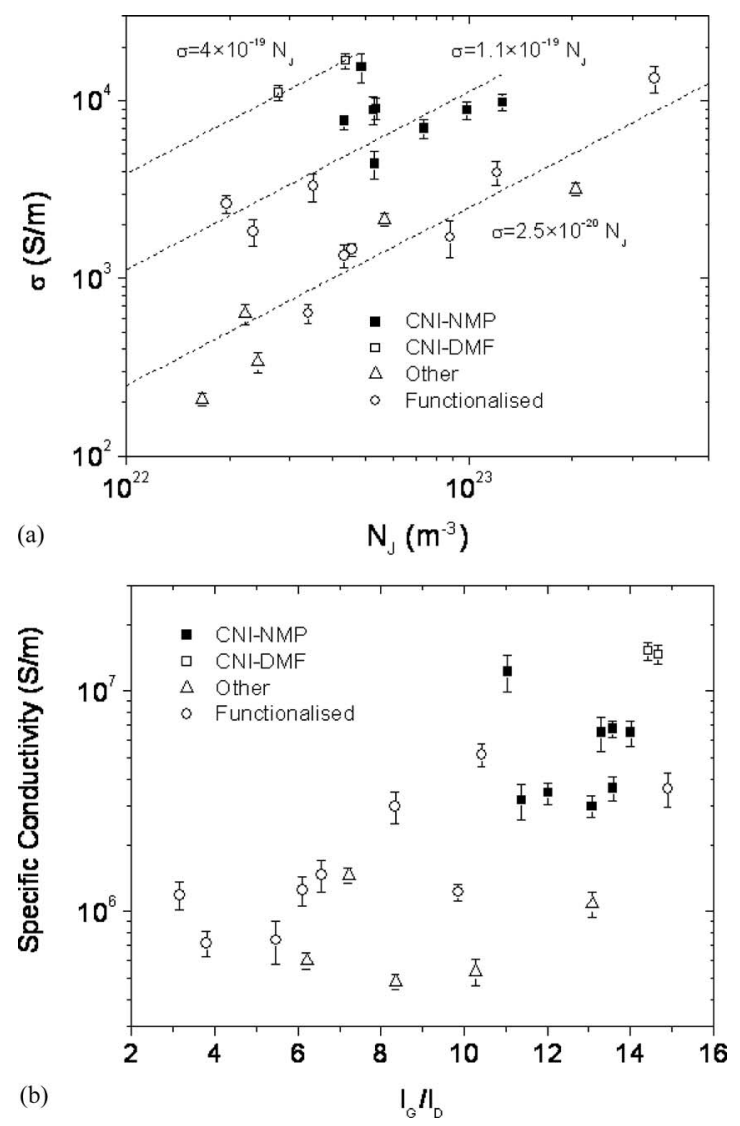

FIG. 5. (a) Approximate scaling of conductivity with number density of inter-NT junctions. The dashed lines illustrate linear scaling as described by the attached equations. (b) Scaling of specific conductivity with the Raman ratio. The specific conductivity is the conductivity rescaled to represent the conductivity of a network produced from the same tubes but with mean bundle diameter $\langle D\rangle=2 \mathrm{~nm}$ and porosity $P=0.5$. This scaling demonstrates that the deviations from linearity in (a) can be correlated to the level of graphitization of the NTs.

Having shown numerically the relationship between network conductivity and junction number density we examine the experimental data for such a relationship. Shown in Fig. 5 (a) is a plot of $\sigma$ versus $\mathrm{N}_{J}$ for each of the tube types which we have studied. Here we observe approximate linear scaling for the CNI-DMF and CNI-NMP NTs. Additionally the majority of functionalized and all of the "other" NTs follow the same approximate linear trend. The dashed lines demonstrate these linear trends. However, two problems are clear: the conductivity of all the functionalized tubes does not scale well with $N_{J}$ and the graph contains significant scatter. These two points will be addressed below.

\section{Effect of intrinsic nanotube properties}

As described above, both modeling and experimental data suggest approximately linear scaling between network conductivity and the junction number density. However this can only occur if all the tubes have the same intrinsic conductivity. With reference to Fig. 3(a), this is not expected to be the case. Thus we need to extract the effect on the film conductivity of variations in the intrinsic NT properties. To do this, we compare the conductivities of all the networks studied in the absence of any morphological differences. We 
do this by combining the linearity of $\sigma$ and $\mathrm{N}_{J}$ with Eq. (4) to rescale the conductivities of each film to represent the conductivity of a film whose porosity and mean bundle diameter are fixed at specific values $P_{0}$ and $D_{0}$. Variations in this parameter are then due to intrinsic differences between tube types. We calculate this specific conductivity $\sigma_{0}$ from

$$
\sigma_{0}=\frac{\sigma}{\mathrm{N}_{J}} \frac{2 \beta}{\pi} \frac{\left(1-P_{0}\right)^{2}}{D_{0}^{3}}
$$

We can also use this method to estimate the maximum realistic conductivity values attainable by appropriate choice of $P$ and $\langle D\rangle$. We do not have much scope to control $P$, thus we chose $P_{0}=0.5$. However, we expect small bundle sizes to lead to conductive films so we chose a realistic minimum mean bundle diameter $\left\langle D_{0}\right\rangle=2 \mathrm{~nm}$. Using Eq. (7), we can calculate the specific conductivity to range from 4.8 $\times 10^{5} \mathrm{~S} / \mathrm{m}$ for Nanocyl SWNT in NMP to $1.5 \times 10^{7} \mathrm{~S} / \mathrm{m}$ for HiPCO dispersed in DMF. It should be pointed out that the upper end of this scale is more than twenty times as high as the most conductive NT film reported to date ${ }^{7}$ suggesting that further improvements on the state of the art are possible.

This allows us to discuss what causes the deviations from linearity, so evident in Fig. 5(a). Looking back to Fig. 3(a), we know that variations in the defect population and hence variation in the NT conductivity result in variations in the film conductivity. This means that when morphological effects are removed, the conductivity should scale with a parameter related to the conductivity of the individual NTs. As we have previously used the Raman ratio $I_{G} / I_{D}$ as a proxy for such a parameter, we plot the specific conductivity versus Raman ratio in Fig. 5(b). Here we see that the HiPCO NT samples are grouped strongly at the top right of the graph indicating high levels of perfection and high intrinsic conductivity. Conversely, for the "other" tube types the specific conductivity is low irrespective of Raman ratio. Most interestingly, the specific conductivity of the functionalized NTs scales exponentially with the Raman ratio. This behavior is most clear for this category because the base tubes are identical with the only variation being the type and degree of functionalization. In general, it is clear that $\sigma_{0}$ scales very strongly with Raman ratio and hence perfection of the NTs. While not surprising, this clearly shows that when morphological effects are removed the NT perfection controls the film conductivity.

This analysis clearly shows that, in addition to depending on the conductivity of the individual NTs, the film conductivity depends on the morphological properties of the network, specifically the junction number density, which can be expressed in terms of $\langle D\rangle$ and $P$. We emphasize that these results are semiempirical and as such are incomplete. For example, Eq. (6) makes no mention of tube length, even though Hecht et $a .^{21}$ showed that film conductivity does indeed scale with tube length. We suggest that a length dependence may be found by a rigorous analysis of the relationship between conductivity and $N_{J}$, i.e., $k=f(L)$. Nevertheless, our data show that high conductivity is achieved for lower porosity films and/or films produced from smaller bundles. This observation also explains why our best films have conductivities an order of magnitude below the best films from the literature. High conductivity films are generally made from ultracentrifuged dispersions that consist mainly of individual NTs and very small bundles. This translates into low values of $D$ and hence high conductivities. In this work we made no attempt to fully exfoliate our NTs resulting in large $D$ values and so lower conductivities.

\section{Estimate of interbundle resistance}

According to Fig. 4(b), the network conductance scales linearly with $N_{J}$. We can write this scaling relationship as $G=2 N_{J} V G_{0} m$, where $m$ is the common slope found in Fig. 4(b). The data in Fig. 4(b) were calculated using a box size of $2 L \times 2 L \times 4 L$ with the electrodes on opposing $2 L \times 2 L$ faces. Taking these dimensions into account we can convert the conductance $G$ to the conductivity $\sigma$ and writing the dimensions in terms of $L$ we get $\sigma=32 m L^{2} G_{0} N_{J}$. We can compare this equation to the experimental data presented in Fig. 5(a). First we note that the parameter $L$ used to define the box size was set as equal to the rod length in all cases. While we do not know the bundle length in the Buckypaper, we know it must be significantly larger than the NT length, which is $\sim 1 \mu \mathrm{m}$ for HiPCO. We assume that $1 \mu \mathrm{m}<L$ $<50 \mu \mathrm{m}$. In the model, the NTs have zero resistance, meaning that the network conductivity was due only to the junctions. This approximation is most valid for the tubes/bundles with the highest intrinsic conductance. Thus, this model is most applicable to nanotube films consisting of either (1) larger bundles that are effectively metallic or (2) individual metallic-only nanotubes. From Fig. 5(b), we can see that the network prepared from HiPCO SWNTs in DMF had the highest intrinsic conductance. From Fig. 5(a), we see that for these tubes $\sigma=4 \times 10^{-19} N_{J}$. Comparing this with the equation above and noting that $m=8 \times 10^{-4}$, from Fig. 5(a) we can estimate the interjunction resistance as $70 \mathrm{k} \Omega<R_{0}$ $<3.5 \mathrm{M} \Omega$. This value is smaller than that estimated by Stahl et $a{ }^{24}{ }^{24}$ for the intertube coupling resistance in NT bundles. This is surprising as we would expect the interbundle junction resistance to exceed the intrabundle tunneling resistance. The reason for the discrepancy is not clear but it is unlikely to be due to our bundle length estimate as we do not expect the bundle length to exceed $50 \mu \mathrm{m}$ in the Bucky paper studied in this work.

\section{E. Parallels with percolation}

Finally we cannot resist noting that the relationship between conductivity and NT volume fraction expressed in Eq. (6) is reminiscent of the standard percolation equation describing the conductivity of conductor-insulator composites ${ }^{25}$ $\sigma=\sigma_{0}\left(V_{f}-V_{f, \text { crit }}\right)^{t}$, where $\sigma_{0}$ is a constant related to the conductivity of the conducting inclusions, ${ }^{26} V_{f, \text { crit }}$ is the critical volume fraction above which the first conducting path through the sample is formed (the percolation threshold), ${ }^{25}$ and $t$ is the critical exponent. ${ }^{25}$ The similarity becomes more marked when one realizes that for NTs, the percolation threshold is generally in the range of $0.1 \%-1 \%^{27-29}$ and so at the high volume fractions studied here, $V_{f}-V_{f \text {,crit }} \approx V_{f}$. More interestingly, in simple systems the critical exponent is 
thought to take the universal value of $t=2.0$ in three dimensions. ${ }^{25}$ Taking these facts into account, the percolation equation becomes identical in form to Eq. (6). However such similarities are deceptive. The percolation equation is only truly valid close to the percolation threshold which is not the case here. In addition, Eq. (6) is derived from consideration of the density of junctions in a dense network, not a percolating system. However, the comparison is instructive nevertheless. Recently, percolation-like behavior with $t=2.2 \pm 0.2$ was reported for very high volume fraction polymer-NT composites $\left(10 \%<V_{f}<45 \%\right) .{ }^{16}$ This was cited as an example of percolationlike scaling, far from the percolation threshold, that is contrary to general expectations. However, our results suggest that the measured exponent may be more indicative of network behavior, described by Eq. (6), than percolation. In fact we can back-calculate the $k$ value associated with the data presented by Blighe et al. ${ }^{16}(\langle D\rangle$ $\left.=13 \mathrm{~nm}, \sigma_{0}=5 \times 10^{4} \mathrm{~S} / \mathrm{m}\right)$ to be $1.1 \times 10^{-19} \mathrm{~S} \mathrm{~m}^{2}$ in good agreement with the range estimated above.

\section{CONCLUSION}

In conclusion we have measured the conductivity of NT films prepared using a large number of different tube types. The films were characterized by Raman spectroscopy, SEM, and density measurements leading to calculation of the Raman $G: D$ ratio, the mean bundle diameter $\langle D\rangle$, and the film porosity $P$. It was found that the conductivity tended to increase in films made from less defective tubes. However the conductivity was lower for films that were either more porous or contained larger bundles. Computer simulations show that the conductivity of a network of conducting rods should scale linearly with the inter-rod junction density. Expressions for the number of junctions per NT and the number density of junctions in terms of the porosity and mean bundle diameter have been derived. Experimentally, reasonable scaling of the conductivity with the junction number density has been observed. This allows the calculation of a specific conductivity, i.e., the expected conductivity for a network of tubes with $P=0.5$ and $\langle D\rangle=2 \mathrm{~nm}$. This specific conductivity tends to scale strongly with Raman ratio. This demonstrates that the properties of both NTs and the network are important for high conductivity films. This work makes it clear that if highly conductive films are required, one needs to work with very conductive, defect-free NTs that have been extensively exfoliated and arranged into dense films. Under these circumstances, our results suggest that conductivities as high as $1.5 \times 10^{7} \mathrm{~S} / \mathrm{m}$ could be achieved.

\section{ACKNOWLEDGMENTS}

We acknowledge Science Foundation Ireland and the Centre for Research on Adaptive Nanostructures and Nanodevices (CRANN) for financial support. We gratefully acknowledge Professor Werner Blau for continuing access to equipment.

${ }^{1}$ C. M. Aguirre, S. Auvray, S. Pigeon, R. Izquierdo, P. Desjardins, and R. Martel, Appl. Phys. Lett. 88, 183104 (2006).

${ }^{2}$ E. Artukovic, M. Kaempgen, D. S. Hecht, S. Roth, and G. GrUner, Nano Lett. 5, 757(2005).

${ }^{3}$ Q. Cao, Z. T. Zhu, M. G. Lemaitre, M. G. Xia, M. Shim, and J. A. Rogers, Appl. Phys. Lett. 88, 113511 (2006)

${ }^{4}$ G. Gruner, J. Mater. Chem. 16, 3533 (2006).

${ }^{5}$ D. H. Zhang, K. Ryu, X. L. Liu, E. Polikarpov, J. Ly, M. E. Tompson, and C. W. Zhou, Nano Lett. 6, 1880 (2006).

${ }^{6}$ A. D. Pasquier, H. E. Unalan, A. Kanwal, S. Miller, and M. Chhowalla, Appl. Phys. Lett. 87, 203511 (2005).

${ }^{7}$ Z. C. Wu, Z. H. Chen, X. Du, J. M. Logan, J. Sippel, M. Nikolou, K. Kamaras, J. R. Reynolds, D. B. Tanner, A. F. Hebard, and A. G. Rinzler, Science 305, 1273 (2004)

${ }^{8}$ L. Hu, D. S. Hecht, and G. Gruner, Nano Lett. 4, 2513 (2004).

${ }^{9}$ B. Ruzicka, L. Degiorgi, R. Gaal, L. Thien-Nga, R. Bacsa, J. P. Salvetat, and L. Forro, Phys. Rev. B 61, R2468 (2000).

${ }^{10}$ H. Z. Geng, K. K. Kim, K. P. So, Y. S. Lee, Y. Chang, and Y. H. Lee, J. Am. Chem. Soc. 129, 7758 (2007).

${ }^{11}$ M. Dressel and G. Gruner, Electrodynamics of Solids: Optical Properties of Electrons in Matter (Cambridge University Press, Cambridge, 2002).

${ }^{12}$ G. Fanchini, H. E. Unalan, and M. Chhowalla, Appl. Phys. Lett. 90, 092114 (2007).

${ }^{13}$ B. B. Parekh, G. Fanchini, G. Eda, and M. Chhowalla, Appl. Phys. Lett. 90, 121913 (2007).

${ }^{14}$ S. D. Bergin, V. Nicolosi, S. Giordani, A. de Gromard, L. Carpenter, W. J. Blau, and J. N. Coleman, Nanotechnology 18, 455705 (2007).

${ }^{15}$ S. Giordani, S. D. Bergin, V. Nicolosi, S. Lebedkin, M. M. Kappes, W. J. Blau, and J. N. Coleman, J. Phys. Chem. B 110, 15708 (2006).

${ }^{16}$ F. Blighe, Y. Hernandez, W. J. Blau, and J. N. Coleman, Adv. Mater. (Weinheim, Ger.) 19, 4443 (2007)

${ }^{17}$ B. Zhao, H. Hu, and R. C. Haddon, Adv. Funct. Mater. 14, 71 (2004).

${ }^{18}$ V. Skakalova, A. B. Kaiser, U. Dettlaff-Weglikowska, K. Hrncarikova, and S. Roth, J. Phys. Chem. B 109, 7174 (2005).

${ }^{19}$ H. Dai, W. Wong, and C. M. Lieber, Science 272, 523 (1996).

${ }^{20}$ C. J. Frizzell, M. in het Panhuis, D. H. Coutinho, K. J. Balkus, A. I. Minett, W. J. Blau, and J. N. Coleman, Phys. Rev. B 72, 245420 (2005).

${ }^{21}$ D. Hecht, L. B. Hu, and G. Gruner, Appl. Phys. Lett. 89, 133112 (2006).

${ }^{22}$ J. Boersma, P. J. de Doelder, and J. K. M. Jansen, SIAM Rev. 25, 102 (1983).

${ }^{23}$ F. M. Blighe, P. E. Lyons, S. De, W. J. Blau, and J. N. Coleman, Carbon 46, 41 (2008).

${ }^{24}$ H. Stahl, J. Appenzeller, B. Lengeler, R. Martel, and P. Avouris, Mater. Sci. Eng., C 15, 291 (2001).

${ }^{25}$ D. Stauffer and A. Aharony, Introduction to Percolation Theory (Taylor \& Francis, London, 1992).

${ }^{26}$ M. Foygel, R. D. Morris, D. Anez, S. French, and V. L. Sobolev, Phys. Rev. B 71, 104201 (2005)

${ }^{27}$ E. J. Garboczi, K. A. Snyder, J. F. Douglas, and M. F. Thorpe, Phys. Rev. E 52, 819 (1995).

${ }^{28}$ D. S. McLachlan, C. Chiteme, W. D. Heiss, and J. J. Wu, Physica B (Amsterdam) 338, 256 (2003).

${ }^{29}$ R. Ramasubramaniam, J. Chen, and H. Y. Liu, Appl. Phys. Lett. 83, 2928 (2003). 